\title{
Impact of a Continuous Quality Improvement Program on Infection Control of Endoscopes
}

\author{
Ahmed Gado ${ }^{1}$ and Basel Ebeid ${ }^{2}$ \\ ${ }^{1}$ Bolak Eldakror Hospital, Giza, Egypt \\ ${ }^{2}$ Banysweef University, Banysweef, Egypt
}

10.3396/ijic.V5i1.010.09

The risk of transmission of infection during gastrointestinal endoscopy is estimated at 1 case per 1.8 million procedures. The reported cases of transmission of infection appear to have resulted from failure to adhere to currently accepted guidelines. ${ }^{1}$

Bolak Eldakror Hospital is a secondary care governmental hospital in Giza, Egypt. The Gastrointestinal endoscopy unit was founded in 1999. A written protocol for endoscope decontamination based on international standards was implemented and an ongoing quality assurance program was established in 2003 when we commenced bacteriological monitoring of endoscopes. Pseudomonas aeruginosa was found in a specimen obtained from a colonoscope. The unit guidelines were reviewed. The cause of contamination was a breach in reprocessing technique. A nurse admitted that she did not remove or clean the cap in the distal tip of the colonoscope at the end of a long busy day. We reprocessed and sampled the colonoscope. The new specimen yielded no growth. The staffing system was changed to secure a balance between the number of cases and work hours. A disinfection logbook and reprocessing checklists were provided for the nurses to record decontamination procedures.

Infection control of endoscopes can be improved by implementing quality improvement activities in routine endoscopy practice.

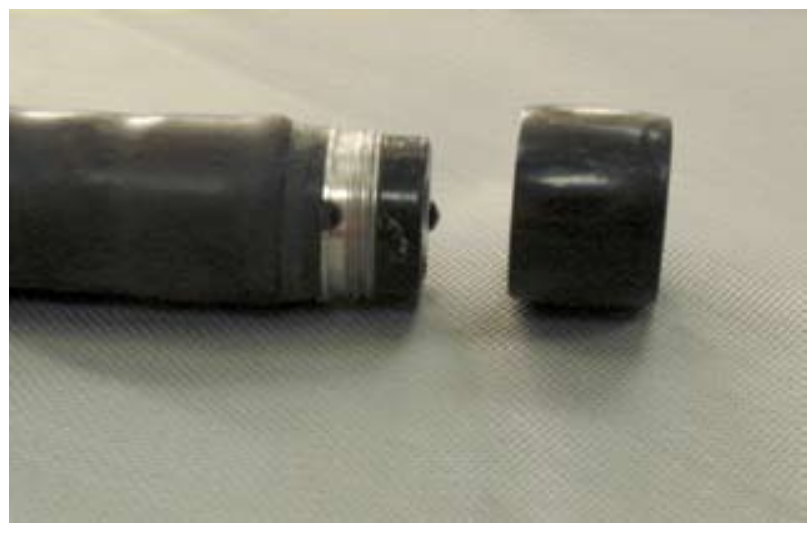

Figure 1. Distal Cap

\section{Reference}

1. Banerjee S, Nelson DB, Dominitz JA, et al. Reprocessing failure. Gastrointest Endosc 2007; 66(5): 869-871. 